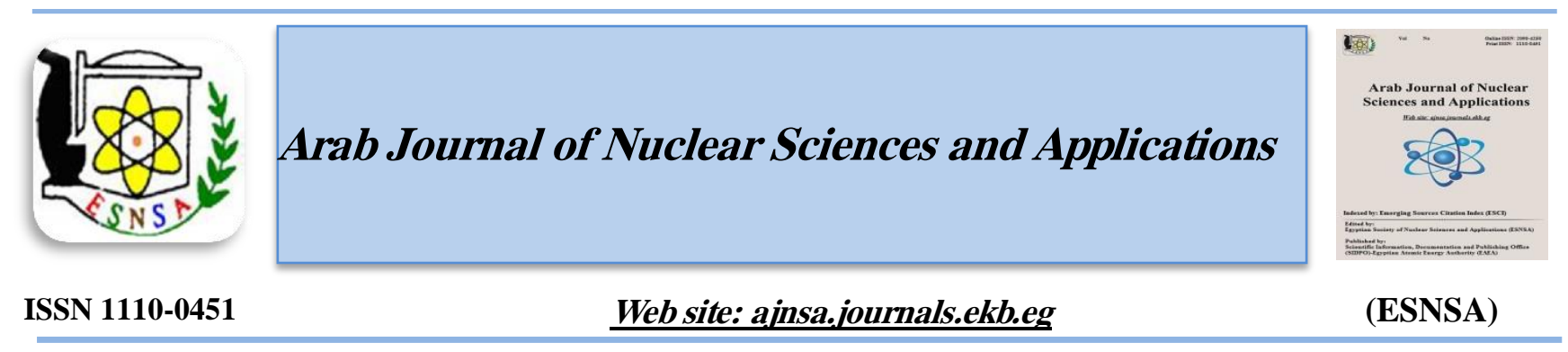

\title{
A Comparative Study of Surface Modification of PS Films Using DC Glow Discharge Plasma in $\mathrm{N}_{2}$ and Ar
}

\author{
O. F. Farag \\ Zagazig University, Faculty of Science, Physics Department, Zagazig, Egypt
}

\begin{abstract}
Received $6^{\text {th }}$ Jun. 2018 In this paper, the polystyrene (PS) film surface was treated using DC glow discharge nitrogen $\left(\mathrm{N}_{2}\right)$ and Accepted $27^{\text {th }}$ Sep. 2018 argon (Ar) plasma to improve the wettability and antibacterial properties. The untreated and plasma treated surfaces were characterized by contact angle, FTIR, XRD and SEM analysis. The antibacterial activity of untreated and plasma treated PS films was evaluated using optical density (OD) technique against Staphylococcus aureus bacteria. The results of the PS film surface treated in both $\mathrm{N}_{2}$ and Ar showed a noticeable increase in the wettability due to the increase in the roughness and the introduction of oxygen-containing polar groups as corroborated with SEM, weight loss calculations and FTIR spectra. The results of antibacterial activity showed that the treated samples inhibit the bacterial growth. The Ar treatment created more oxygen-containing polar groups and increase roughness on the PS film surface and extensively modified the polymer surface than the $\mathbf{N}_{2}$ treatment.
\end{abstract}

Keywords: DC plasma; Surface modification; Polymer film; Hydrophilicity; Antibacterial activity

\section{Introduction}

Polystyrene (PS) is one of the most widely used polymers today, it has a wide range of applications such as foam packaging, kitchen appliances, toys, drinking cups, computer industry, surgical and domestic instruments [1-3]. Also, PS has excellent physical properties and low cost [4]. Despite these excellent properties, PS is rigid and hydrophobic polymer [5]. Certain applications such as cell culture discs [6] required hydrophilic surface, without affecting the good bulk properties of the polymers. According to statistical data from the American Disease Control and Prevention Center, from each 35 million patients, 1.8 million patients in America suffer from bacterial infection caused by medical polymer tubes. Consequently, this limits the use of PS in medical applications. To overcome these limitations, surface modification techniques have been used in the last decades to improve the hydrophilicity and to give PS antibacterial activity [3].

There are various surface modification techniques such as corona treatment, UV, flame, gamma ray, ion beam, electron beam irradiations, laser treatments and glow discharge plasma treatment [7-11]. Among these techniques, glow discharge plasma treatment technique is considered to be the most promising method for the modification of the surface properties of polymeric materials. Glow discharge plasmas contain charged and neutral particles, such as electrons, ions, atoms, molecules and radicals. Depending on the gas composition and treatment conditions, the energetic plasma species (electrons, ions, fast atoms, free radicals and UV photons) participate in polymer surface treatment, resulting in three main effects:(i) etching, (ii) activation and (iii) cross-linking [12]. The advantages of this technique is that plasma treatment changes the properties of the material

Corresponding author: FaragFarag66@yahoo.com

DOI: $10.21608 / a j n s a .2019 .23358$

(C) Scientific Information, Documentation and Publishing Office (SIDPO)-EAEA 
only a thin near-surface layer typically with depth 0.005 to $0.05 \mu \mathrm{m}$ [13]. In addition, it is a rapid and environmentally friendly process [14].

The effect of plasma treatment depends on a verity of parameters such as type of plasma (DC, radio frequency (RF) or microwave (MW)), the plasma power, the pressure of the working gas, flow rate or gas mixture (type of working gas), as well as treatment time [15]. In the present study, PS films were treated by DC glow discharge plasma with two different working gases $\left(\mathrm{Ar}\right.$ and $\mathrm{N}_{2}$ ) at low pressure to improve the hydrophilicity and antibacterial activity properties of the films. To evaluate the extent of the modifications by the two different working gases $\left(\mathrm{Ar}\right.$ and $\mathrm{N}_{2}$ ), the untreated and plasma treated samples were investigated by contact angle measurements, SEM, XRD and FTIR analyses. The antibacterial activity of the modified films was investigated using optical density (OD) technique.

\section{Materials and Methods}

\section{Samples preparation}

The studied PS samples were fabricated by the casting method. Polystyrene (PS) of molecular weight of 135,000 in the form of grains and toluene with purity of $99.998 \%$ were supplied by
Sigma-Aldrich company. The desired amount of PS was dissolved in $20 \mathrm{ml}$ toluene and the mixture was stirred using a magnetic stirrer at room temperature for one hour to get the complete dissolution. The obtained solution was then poured into a clean glass Petri dish. The evaporation of toluene was carried out by placing the samples in a dust free chamber at room temperature for one week. The films thickness was in the range of 0.22-0.25 mm, thickness was determined using a digital micrometer at different places in each film and an average thickness was taken.

\section{Plasma treatment}

Figure (1) presents a schematic diagram of the DC plasma unit used for surface treatment of the PS films and its details were described in previous studies [16]. The samples were inserted inside the plasma reactor at the interface between the cathode fall region and the negative glow region and supporting them on a glass rode. The treatment conditions in both $\mathrm{N}_{2}$ and Ar were kept constant, as the working gas pressure was 0.4 Torr, the plasma power was about $3.5 \mathrm{~W}$ and the treatment time was varied from 15 to 60 minutes. The size of the treated samples was $(1 \times 2 \mathrm{~cm})$.

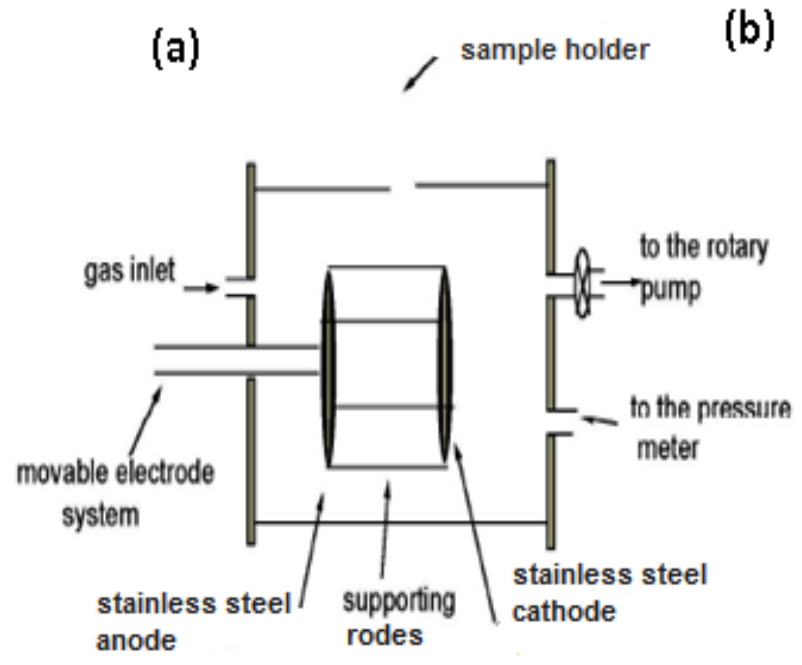

(b)

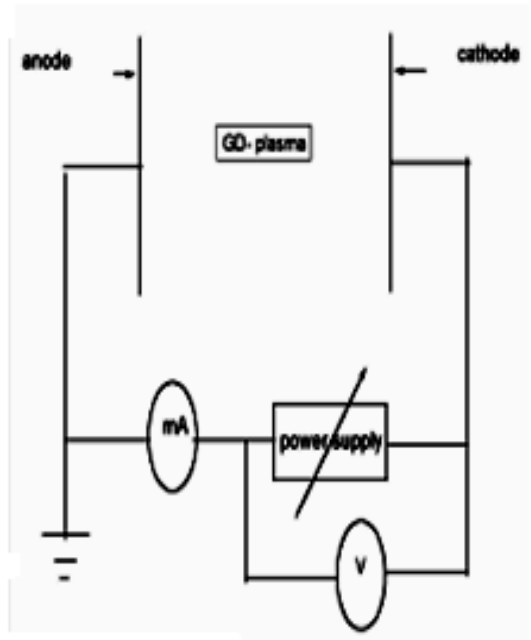

Fig. (1): A schematic dagran for (a) The experimental equipment and (b) The electrical circuit 


\section{Characterization techniques}

\section{Scanning electron microscope (SEM)}

For surface morphology investigation, the scanning electron microscope quanta fei 250 , was used to reveal images of the surfaces before and after treatment with $\mathrm{N}_{2}$ and Ar plasmas.

\section{Weight loss analysis}

In order to quantify the plasma-etching effects on the surface of PS films, a microbalance was used to measure the weight of the samples before and after the plasma treatment. The plasma-etching effect in terms of weight loss was calculated by the following equation [17]:

$$
\operatorname{Weightloss}(\%)=\left[\frac{\left(W_{p}-W_{p t}\right)}{\left(W_{p}\right)}\right] \times 100
$$

Where $W_{\mathrm{p}}$ and $W_{\mathrm{pt}}$ are the weight of pristine and plasma treated samples, respectively.

The surface roughness of a solid surface plays an important role in the wettability property of the surface. Wenzel [18] was the first one who discovered the effect of surface roughness on the wettability property (contact angle) of a surface. For the contact angle on a rough surface, the surface roughness was calculated using the following equation [18]:

$$
R=\left[\frac{\operatorname{Cos}\left(\theta_{p t}\right)}{\operatorname{Cos}\left(\theta_{p}\right)}\right]
$$

Where $\theta_{\mathrm{p}}$ and $\theta_{\mathrm{pt}}$ are the contact angles of pristine and plasma treated film surfaces, respectively.

\section{Fourier transform infrared (FTIR) spectroscopy}

The FTIR spectra were taken for pristine and plasma treated PS samples to determine the chemical functional groups which may be formed on the surface of the PS films upon plasma treatment. The samples were examined using an infrared spectrometer device, Vertex 70 Bruker Optics.

\section{$X$-ray diffraction (XRD)}

To investigate the crystalline structure of untreated and plasma treated PS films, the XRD patterns were taken using a Bruker-AXS D8 advanced diffractometer in the $2 \square$ range $\left(5^{0}-80^{\circ}\right)$. X-ray tube with $\mathrm{CU}$ anode $\left(\lambda=1.5406 \mathrm{~A}^{0}\right)$ was operated at $40 \mathrm{kV}$ and $40 \mathrm{~mA}$.

\section{Contact angle measurements}

The improvement in the hydrophilicity of the PS films was examined by measuring the water contact angle using a travelling microscope. Measurements were performed with deionized water at room temperature. The volume of the deionized water drop was about $5 \mu$ using a microsyringe. Measurements were repeated eight to ten times at different points on the surface of the same PS sample and the average value was taken.

\section{Antibacterial properties of PS films}

The antibacterial activity of untreated and plasma treated PS films against Staphylococcus aureus bacteria as Gram-positive bacteria were examined by applying the optical density (OD) technique as described elsewhere [19]. The $\mathrm{N}_{2}$ and Ar treated samples and the untreated samples were inserted in bacterial suspensions that were incubated at $37{ }^{\circ} \mathrm{C}$ for $24 \mathrm{~h}$. After incubation, the washing process of PS samples was carried out with $20 \mathrm{ml}$ of $0.87 \%$ $\mathrm{NaCl}$ solution containing polysorbate 80 at $\mathrm{pH} 7$ [20]. Afterwards, the OD of growth was measured in a spectrophotometer at $\lambda=600 \mathrm{~nm}$ to assessed the growth of the bacteria and its adherence to PS films.

\section{Results and Discussion Plasma-etching effect}

The removing process of low-molecular contaminants such as additives, processing acids, and adsorbed species due to the interaction of active plasma species (ions, electrons, radicals, and UV photons) with the surface of polymer films is known as plasma etching. After plasma etching, ablation of polymer chain starts. This is due to the breaking up of bonds, chain scission, and the processes of degradation [21]. This causes a loss in the weight of the film and alters the surface morphology resulting in an enhancement in wettability of the treated samples.

Figure (2) displays the weight loss $\%$ as a function of the treatment time for the plasma treated PS films using nitrogen and argon. It is obvious that by increasing the treatment time, the weight loss \% of the PS surface was increased in both nitrogen and argon plasma treatments. The weight loss \% for the Ar plasma-treated PS surface was higher than for the $\mathrm{N}_{2}$ plasma-treated PS surface. This can be explained as follows: the etching process during plasma treatment depends on the density of active plasma species bombardment towards the surface. Argon plasma contains more electrons and ions than in the case of the nitrogen plasma [16].This results in more bond scission in the argon plasma treatment and consequently more weight loss. 


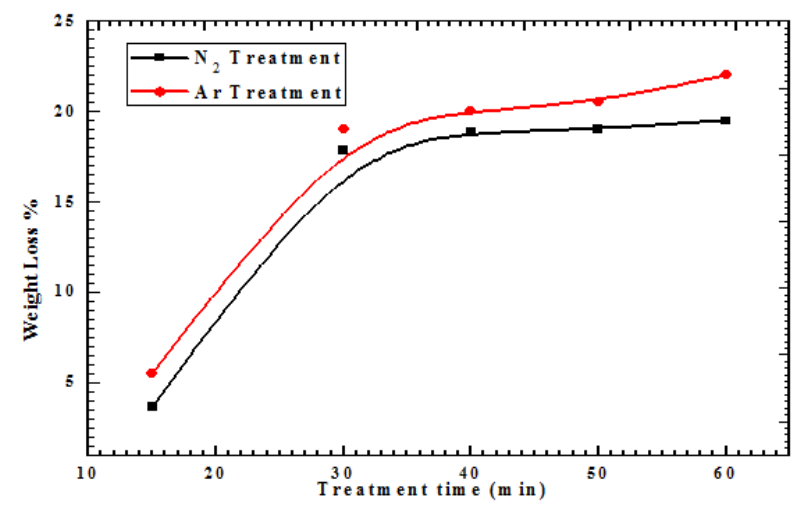

Fig. (2) Weight loss \% of plasma-treated PS films as a function of the treatment time

To investigate the plasma the etching effect on the surface morphology of PS films, SEM was performed for the untreated and plasma-treated samples. Figure (3) presents the SEM images for (a) the untreated and (b) and (c) for plasma-treated films under nitrogen and argon with treatment time one hour, respectively. By comparing Figure (3a) with (3b) and (3c), it is clearly noticed that plasma treatment changes the surface morphology of the PS films. Figure (3) demonstrates that the untreated PS surface is smooth, while many pores and scratches can be observed on the PS surface after the plasma treatment. The appearance of these pores and scratches on the PS surface after the plasma treatment increase surface roughness, which, in turn, enhance the wettability of the plasma treated samples [22]. Also, it is observed that Ar treatment alters the surface morphology of the PS film more than $\mathrm{N}_{2}$ treatment.

The surface roughness (R) of the PS films was determined using the measured values of deionized water contact angle according to equation 2 . Figure (4) displays the surface roughness as a function of the treatment time for the plasma treated PS films under nitrogen and argon. It can be seen that by increasing the treatment time, the surface roughness of the PS surface was increased in both nitrogen and argon plasma treatment. Also, the surface roughness for the Ar plasma-treated PS surface was higher than for the $\mathrm{N}_{2}$ plasma-treated PS surface. The increase in the surface roughness with the increase of the treatment time is an indication of the enhancement in the wettability of plasma-treated samples.
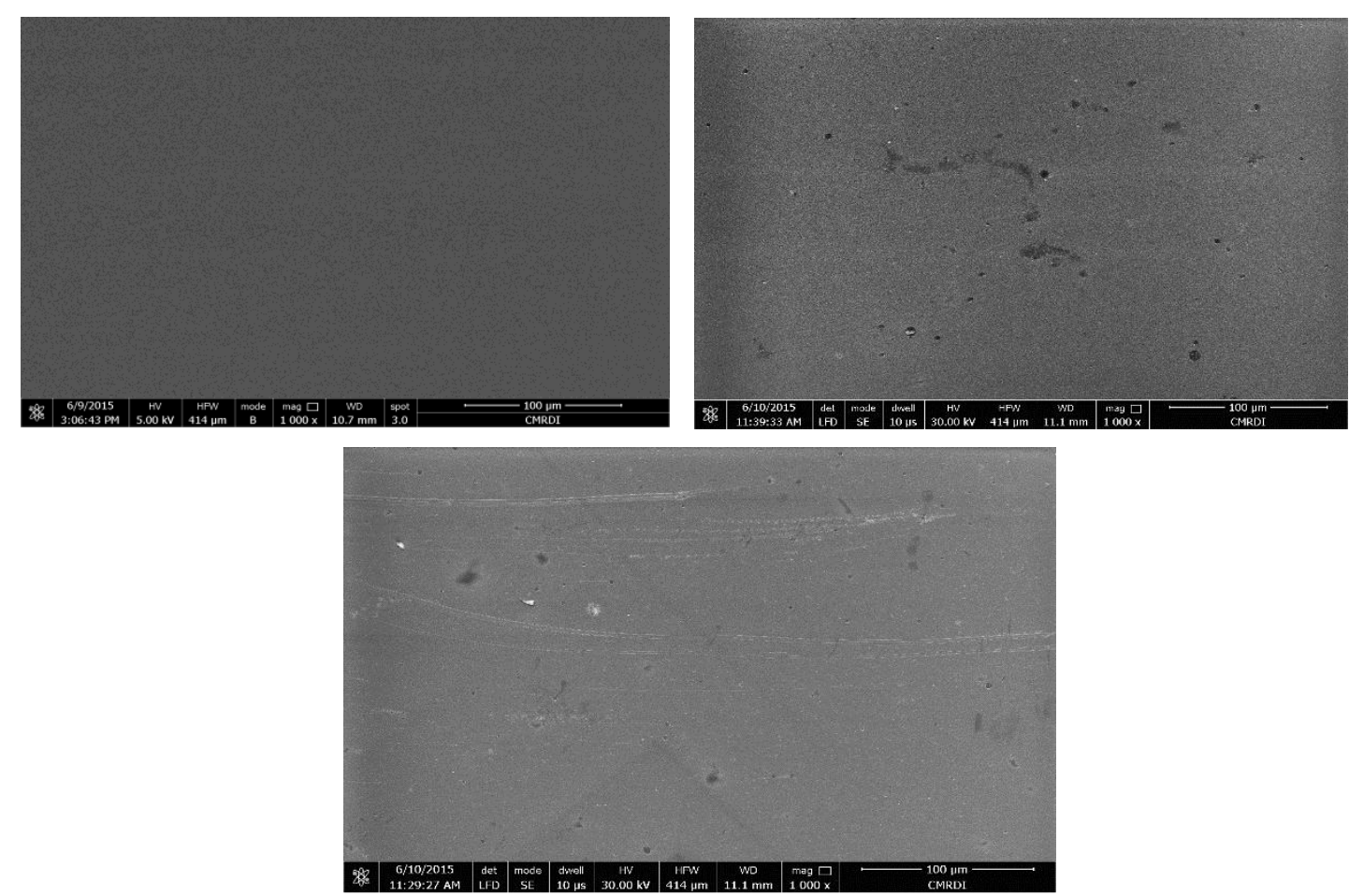

Fig. (3): SEM micrograph of untreated and plasma treated PS film surfaces (a) untreated (b) $\mathbf{N}_{2}$ plasma treated (c) Ar plasma treated PS. 


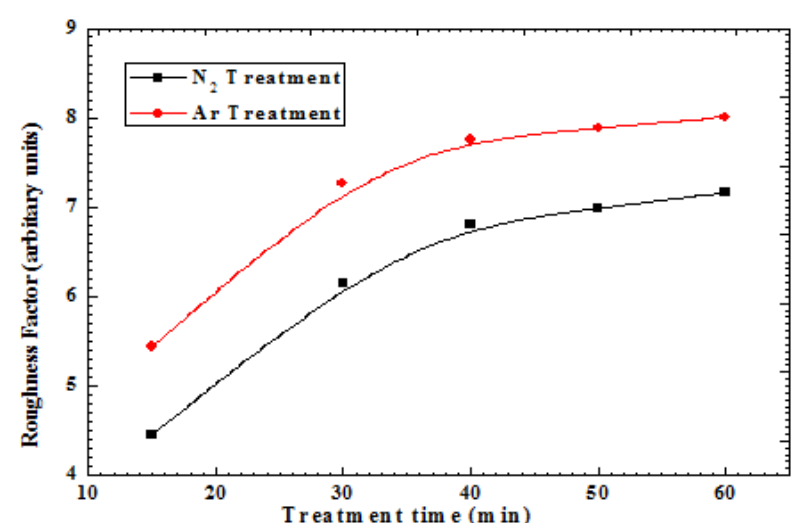

Fig. (4): Variation of surface roughness with the treatment time for N2 and Ar plasma-treated PS films

\section{Structural analysis}

\section{$X R D$ results}

$\mathrm{XRD}$ is a useful analysis technique used to investigate the effect of plasma treatment on the crystalline structure and bulk properties of PS films. Figure (5a), displays the XRD spectra for the untreated and nitrogen plasma-treated PS film for different treatment times. The figure clearly reveals that PS film is amorphous in nature since the diffraction pattern is characterized by halos (humps) extending over the $2 \theta$ range $10^{\circ}$ to $24^{0}$ [23]. It is clearly observed that the only significant difference between the untreated and nitrogen plasma-treated samples is reflected in the intensity of the diffraction peaks. Increasing the treatment time increase the intensity of diffraction peaks in systematic manner.
Figure (5 b) shows the XRD spectra for plasmatreated PS film under nitrogen and argon for treatment time of one hour. It is clearly observed that the diffraction peaks of Ar plasma- treated sample were more intense than those treated samples under nitrogen. Hence, the Ar plasma treatment increases the crystalline nature of the PS film than the $\mathrm{N}_{2}$ plasma treated one. The obtained results of XRD confirm that the plasma treatment improves the crystalline nature of PS film surface without affecting the bulk properties [12].

\section{FTIR analysis}

In order to identify the formation of new chemical functional groups on the surfaces due to plasma treatment, the FTIR was employed. Figure (6) shows FTIR-spectra for the pristine sample and $\mathrm{N}_{2}$ plasma treated samples for different treatment times. As obviously demonstrated in Fig. 6, the main characteristics absorption bands of PS are five peaks over the $2800-3100 \mathrm{~cm}^{-1}$ range, assigned to the stretching mode of $\mathrm{C}-\mathrm{H}$ bonds in the main chain and benzene rings, and the peaks at 1601, $1492,1451,1027,757$ and $698 \mathrm{~cm}^{-1}$ are identified as the deformation and skeletal vibrations of $\mathrm{C}-\mathrm{H}$ bonds in PS [23]. By comparing the FTIR spectra of $\mathrm{N}_{2}$ plasma treated PS samples with the pristine sample, it is obvious that the only difference between them is in the appearance of a broad peak in the range $3200-3700 \mathrm{~cm}^{-1}$ which was observed for all the samples. This region belongs to hydroxyl group (-OH) [24]. Also, increasing the treatment time increases the intensity of the hydroxyl group.
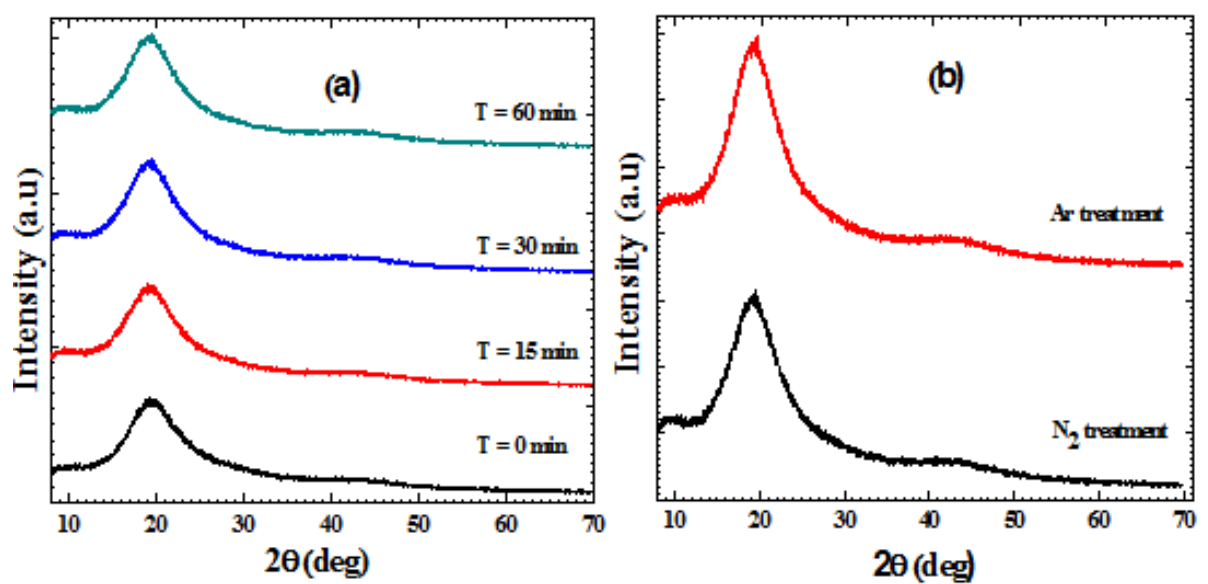

Fig. (5):X-ray diffraction spectra for untreated and plasma treated PS films (a) under N2 at different treatment times and (b) under $\mathrm{N} 2$ and $\mathrm{Ar}$ at treatment time $60 \mathrm{~min}$ 

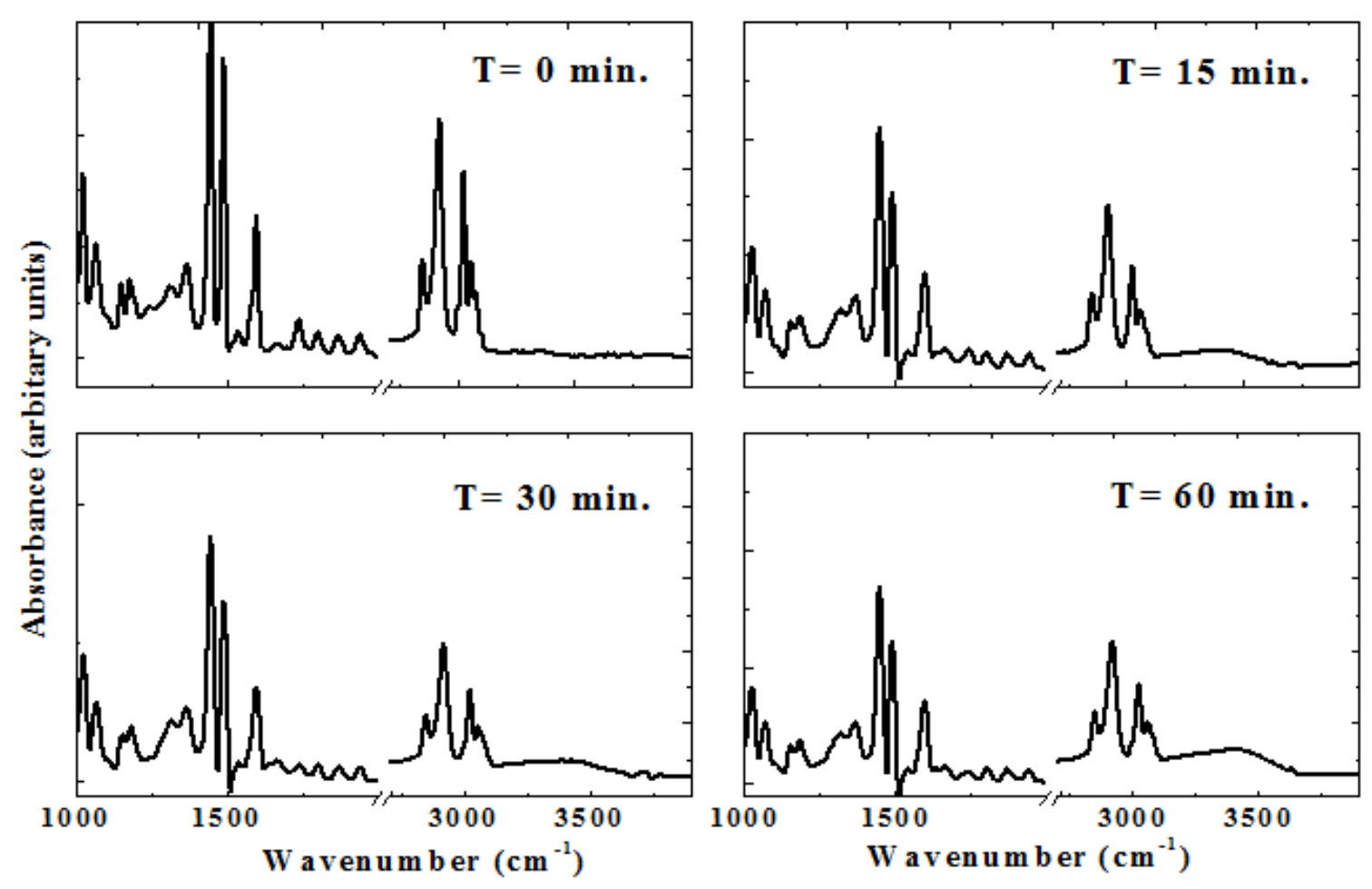

Fig. (6): FTIR spectra for untreated and N2 plasma treated PS films at different treatment times

Figure (7) shows the FTIR-spectra of plasma treated PS samples under $\mathrm{N}_{2}$ and Ar for treatment time one hour. It has been found that the remarkable difference between them is in the intensities of the main characteristics absorption bands of PS and of the broad peak. The Ar treatment reduces the intensities of the main characteristics absorption bands of PS than $\mathrm{N}_{2}$ plasma treatment. This may be attributed to high etching rate of Ar than $\mathrm{N}_{2}$. Also, the intensity of ($\mathrm{OH})$ in Ar treated samples is higher than $\mathrm{N}_{2}$ treated samples. This explains the higher efficiency of $\mathrm{Ar}$ treatment in enhancing the wettability of PS films than $\mathrm{N}_{2}$ treatment.

The incorporation of $\mathrm{O}_{2}$ functionalities on the sample's surfaces is an indication of surface activation by $\mathrm{N}_{2}$ and Ar plasma treatments and the formation of free radicals site on the PS film's surfaces. The activated surface either adsorbs moisture from ambient air just after removing from the plasma chamber or abstract residual $\mathrm{O}_{2}$ in the plasma chamber [25]. The formation of hydroxyl functional groups on the surface of plasma-treated samples is the main reason of wettability enhancement
The extent of the surface hydrophilicity (wettability) improvement of plasma treated PS films was evaluated by measuring the surface contact angle. Figure 8 displays the variation in the deionized water contact angle of the plasma treated PS films for different treatment times in $\mathrm{N}_{2}$ and $\mathrm{Ar}$ plasmas. It can be noticed that the deionized water contact angle for the pristine PS film is $83^{\circ}$. As the treatment time increases the deionized water contact angle decreases in both $\mathrm{N}_{2}$ and $\mathrm{Ar}$ treatments. The noticed enhancement in the wettability of PS films due to $\mathrm{N}_{2}$ and Ar treatments was attributed to the formation of oxygen containing groups and the increase in surface roughness which have been confirmed by FTIR and SEM analysis in the above section [26]. Also, it is clear that the Ar plasma treatment is more efficient than $\mathrm{N}_{2}$ plasma treatment in enhancing the wettability of the PS films. This may be attributed to the higher plasma density and low energy of electrons or ions in Ar plasma than $\mathrm{N}_{2}$ plasma at the same treatment conditions [16].

\section{Antibacterial properties}

The antibacterial activity analysis for PS films was carried out for pristine and plasma-treated samples. The experiment was performed against 
Staphylococcus aureus bacteria and analyzed by the optical density (OD) technique. The results in Table 1 reveal that the growth of tested $S$. aureus was decreased by increasing of plasma treatment time compared with the untreated one. The highest inhibition was obtained after a treatment time of 30 min. for both $\mathrm{N}_{2}$ and Ar treatments compared with the untreated samples. The antibacterial activity of PS films decreased by further increasing the treatment time up to $60 \mathrm{~min}$., but still lower than the untreated one. The antibacterial activity (the inhibition of bacterial growth) of plasma-treated PS films could be attributed to the introduction of oxygen containing groups such as (-OH) group onto the surface of PS by $\mathrm{N}_{2}$ and Ar treatments as confirmed from FTIR analysis [27,28]. Also, the results of optical density of growth indicated a higher antibacterial activity of the treated PS samples with Ar plasma than $\mathrm{N}_{2}$-treated samples. This could be attributed to Ar plasma incorporating more hydrophilic (oxygen containing) groups onto the surface of PS than $\mathrm{N}_{2}$ plasma, which contributes to the enhancing in wettability and antibacterial activity properties.

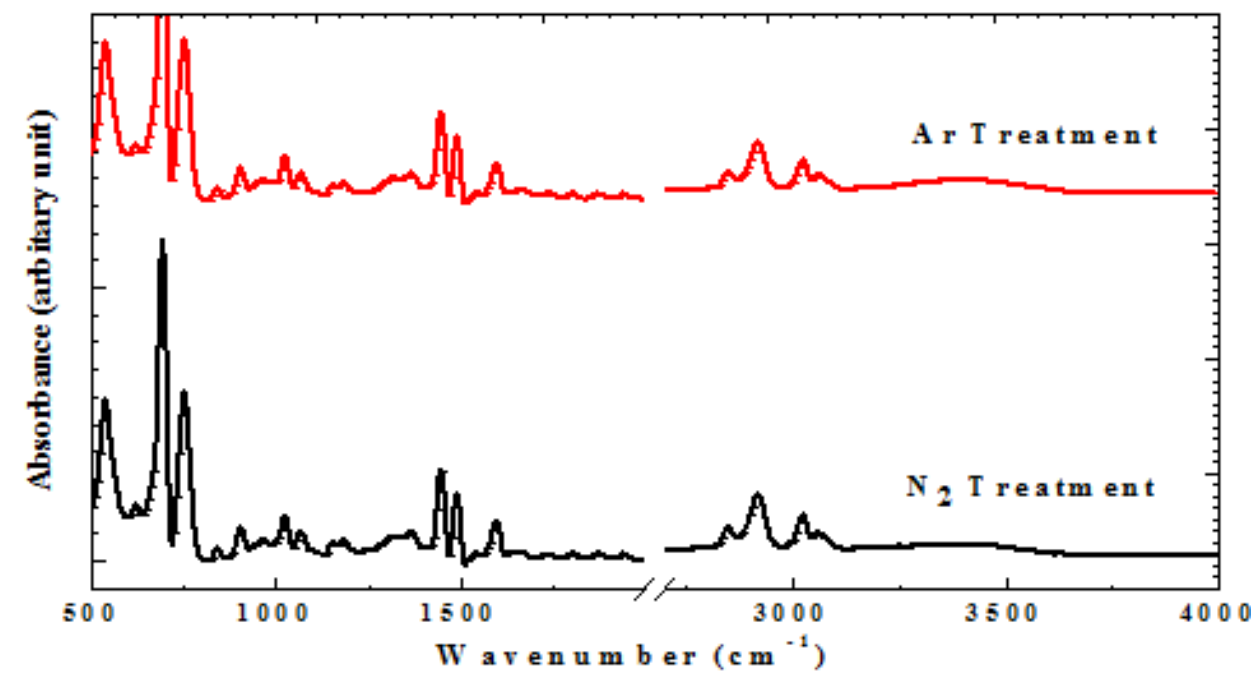

Fig. (7): FTIR spectra for plasma treated PS films under N2 and Ar at treatment time 60 min

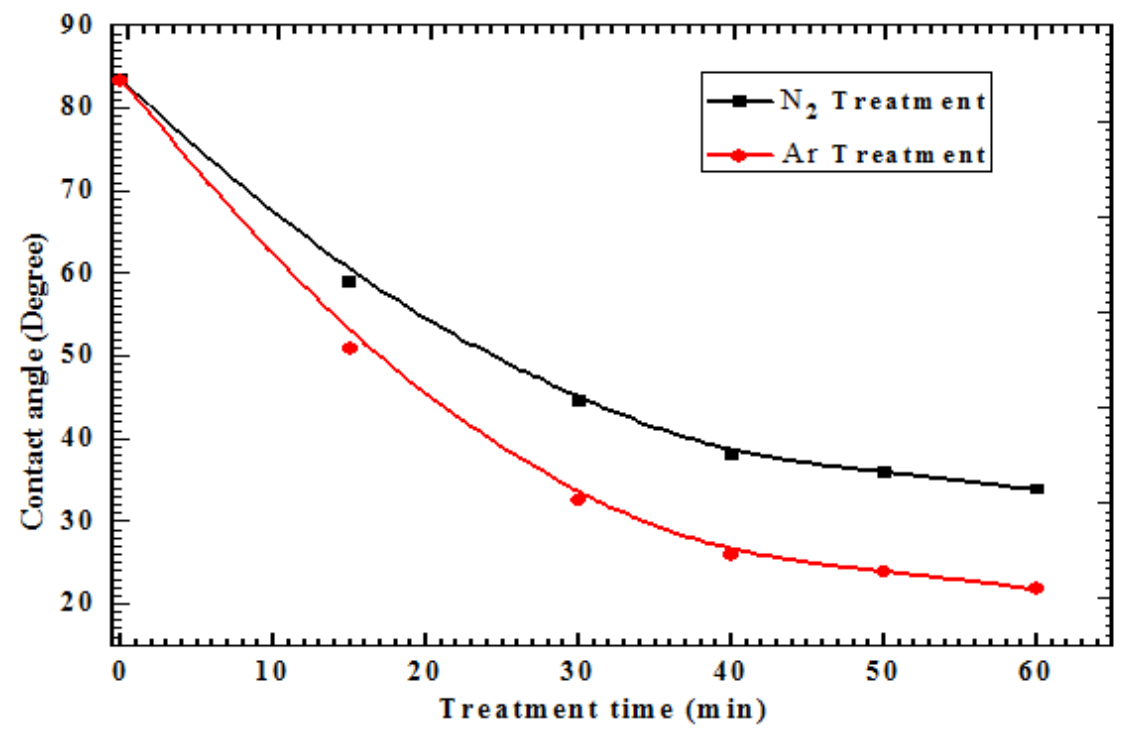

Fig. (8) Variation of the water contact angle of untreated and plasma treated PS films at different treatment times under N2 and Ar 
Table (1): Effect of $\mathrm{N}_{2}$ and Ar plasma treatments at different exposure times on growth of $S$. aureus 15 strains

\begin{tabular}{c|c|c}
\hline \multirow{2}{*}{ Treatment time (min.) } & \multicolumn{2}{|c}{ Optical Density $($ OD) $($ at 600nm) } \\
\cline { 2 - 3 } & $\mathrm{N}_{2}$ Treatment & Ar Treatment \\
\hline 15 & 0.459 & 0.459 \\
30 & 0.420 & 0.389 \\
40 & 0.409 & 0.365 \\
50 & 0.410 & 0.375 \\
60 & 0.411 & 0.379 \\
\hline
\end{tabular}

\section{Conclusion}

DC glow discharge $\mathrm{N}_{2}$ and Ar plasma was employed to improve the surface wettability and antibacterial properties of PS films. The enhancement in the surface wettability of the plasma treated samples is confirmed by FTIR spectroscopy, contact angle studies and SEM analysis. The results of optical density (OD) of growth confirm the antibacterial activity of plasma treated samples. Finally, the Ar plasma-treatment enhances the surface wettability and antibacterial properties of PS films more than $\mathrm{N}_{2}$ plasmatreatment.

\section{References}

1- M. S. Sheu, D. M. Hudson, and I. H. Loh, "Encyclopedia hand book of biomaterials and bioeng, part A", Marchel Dekker, New York, (1995).

2- C. M. Chan, and T. M. Ko; Surf. Sci. reports; 24, 1 (1996).

3- S. M. Pawde, and S. S. parab; Pramana J. Physc.; 79, 935 (2008).

4- S. T. Rabie, A. M. Mahran, E. M. Kamel, and Abdel N. H. Hamid; J. Appl. Scin. Res.; 4, 2015 (2008).

5- T. J. Alwan, M. P. J.; 5, 204 (2010).

6- R. I. Freshney, "in Culture of Animal Cells", $3^{\text {rd }}$ edition, Wiley, New York, (1994).

7- R. K. Y. Fu, I. T. L. Cheung, Y. F. Mei, C. H. Shek, G. G. Siu, P. K. Chu, W. M. Yang, Y. X. Leng, Y. X. Huang, X. B. Tian, and S. Q. Yang; Nucl. Instrum. Methods Phys. Res., Sect. B; 237, 417 (2005).

8- H. Kaczmarek, and H. Chaberska; Appl. Surf. Sci.; 252, 8185 (2006).

9- M. Ozdemir, C. U. Yurteri, and H. Sadikoglu; Crit. Rev. Food Sci. Nutr.; 39, 457 (1999).
10- W. Zhang, P. K. Chu, J. Ji, Y. Zhang, and Z. Jiang; Appl. Surf. Sci.; 252, 7884 (2006).

11- E. O. Filippova, D. A. Karpov, A. V. Gradoboev, V. V. Sokhoreva, and V. F. Pichugin; ISSN 2075-1133, Inorganic Materials: Applied Research; 7, 664 (2016)

12- K. N. Pandiyaraj, V. Selvarajan, R. R. Deshmukh, and M. Bousmina; Surf. Coat. Technol.; 202, 4218 (2008).

13- S. H. Mortazavi, M. Ghoranneviss, and A. H. Sari; J. Fusion Energ.; 30, 48 (2011)

14- J. Yip, K. Chan, K. M. Sin, and K. S. Lau; J. Mater. Process. Technol.; 123, 5 (2002).

15- S. Kitova, M. Minchev, and G. Danev; J. Optoelectronics and Advanced materials; 7, 249 (2005).

16- M. M. Mansour, N. M. El-Sayed, O. F. Farag and M. H. Elghazaly; Arab J. Nucl. Sci. Appl.; 46, 116 (2013).

17- K. N. Pandiyaraj, V. Selvarajan, R. R. Deshmukh, C. Gao; Vacuum; 83, 332 ( 2008).

18- K. L. Mittal, "contact angle, wettability and adhesion", $3^{\text {rd }}$ edition, Taylor \& Francis, The Netherlands, (2003).

19- N. M. EL-Sayed , F. M. Reda , O. F. Farag, and D. A. Nasrallah; J. Biol. Phys.; 43, 211(2017).

20- K . N. Pandiyaraj, R. R. Deshmukh, B. S Ram, and R. Mahendiran; J. Nanosci. Nanotechnol.; 2, 436 (2014).

21- P. Blais, D. J. Cartsson, and D. M. Wiles; J. Poly. Sci.;10, 1077 (1972).

22- M. R. Sanchis, O. Calvo, O. Fenollar, D. Garcia, and R. Balart; Polymer Testing; 27, 75(2008).

23- B. Jaleh. M. S. Madad, M. F. Tabrizi, S. Habibi, R. Golbedaghi, and M. R. Keymanesh; J. Iran. Chem. Soc.; 8, S161(2011).

Arab J. Nucl. Sci. \& Applic. Vol. 52, No. 1 (2019) 
24- N. M. Elsayed, O. F. Farag, M. H. Elghazaly, D. A. Nasrallah; IOSR- J PT E; 3, 36 (2016).

25- S. B. Idage and S. Badrinarayanan; Langmuir ;14, 2780 (1998).
26- H. Mirzadeh, and Sh. Bagheri; Rad. Phys. Chem.; 76, 1435 (2007).

27- K. N. Pandiyaraj, R. R. Deshmukh, R. Mahendiran, Pi-G Su, E. Yassitepe, I. Sah, S. Perni, P. Prokopovich and M. N. Nadagouda; Mat. Sci. Eng. C, 36, 309 (2014).

28- P. Anuradha, and A. A. Kalianib; Int. J. Pure Appl. Phys., 5, 157 (2009). 